\title{
Orthokeratinized Odontogenic Cyst: A Rarity
}

\author{
Heena Sonawane, Freny R Karjodkar, Kaustubh Sansare, Nimish Prakash
}

\begin{abstract}
Orthokeratinized odontogenic cyst (OOC) was first identified as the rare variant of keratocystic odontogenic tumor (KCOT) for its different histopathology and rare recurrence which was reclassified by WHO in 2005. The orthokeratinized odontogenic cyst is a distinct clinicopathologic entity and is histologically characterized by a thin, uniform, epithelial lining with orthokeratinization and a subjacent granular cell layer. The basal cells are usually cuboidal or flattened. OOC in maxilla is rare. This article presents a case of 56-years-old male patient with OOC in left maxilla. The clinical, radiographic and histological features of the cyst are discussed in this case report.
\end{abstract}

Keywords: Maxilla, Orthokeratinized odontogenic cyst, Keratocystic odontogenic tumor.

How to cite this article: Sonawane H, Karjodkar FR, Sansare K, Prakash N. Orthokeratinized Odontogenic Cyst: A Rarity. Int J Otorhinolaryngol Clin 2013;5(3):182-186.

Source of support: Nil

Conflict of interest: None declared

\section{INTRODUCTION}

Odontogenic keratocyst (OKC) was described by Philipsen in $1956^{1}$ with more aggressive clinical behavior, including a high recurrence rate, high mitotic count and a high epithelial turnover. Hence WHO reclassified OKC as keratocystic odontogenic tumor in 2005. ${ }^{2}$ Although both the first two editions of the World Health Organization's (WHO) histological classification of odontogenic tumors recognized that orthokeratosis was found in some cases, ${ }^{3}$ the WHO's 2005 edition expressly excluded it from its definition of a KCOT. The 2005 edition reclassified the parakeratotic type as a keratocystic odontogenic tumor (KCOT) and stated 'Cystic jaw lesions that are lined by orthokeratinizing epithelium do not form part of the spectrum of a KCOT' ${ }^{4}$ A main feature that persuaded Wright ${ }^{5}$ to consider orthokeratinized odontogenic cyst (OOC) as a separate entity, other than the histopathology, was the reduced rate of recurrence. The OOC was also almost absent from cases of 'nevoid basal cell carcinoma syndrome'; only Bolbaron et al have reported one such case. ${ }^{5,6}$ OOCs displaced adjacent teeth and root resorption was not a feature of the OOC and this differs markedly from KCOT. ${ }^{7}$ The visually sriking feature of OOCs was their buccolingual expansion.

\section{CASE REPORT}

A 56 years old male patient reported to the Department of Oral Medicine Diagnosis and Radiology, Nair Hospital Dental College, with the chief complain of swelling in the upper left anterior region of the jaw since 4 months. The patient gave history of trauma 8 years back in relation to left central incisor and extraction of maxillary left 2nd premolar and 2nd molar, 5 months back causing the swelling to regress, which reappeared within 15 days of extraction. Patient presented a histological report of the incisional biopsy which gave an impression of an infected keratocyst. Extraoral examination revealed a hard tender swelling on the maxillary left anterior region causing obliteration of the nasolabial fold and mild facial asymmetry (Fig. 1). Intraoral examination presented a hard tender swelling seen on the maxillary left palatal region crossing the midline extending from maxillary right left central incisor to the maxillary left 1st molar involving most of the left palatal anterior region. Maxillary left 2nd premolar and 2nd molar were missing. Teeth displacement was noted in relation to maxillary right lateral incisor and canine and maxillary left canine (Fig. 2). After correlating the clinical findings, the most probable diagnosis considered was KCOT, and the differential diagnosis was OOC.

Then the patient was scheduled for various radiographs, such as orthopantomogram and computed tomography (CT scan).

Panoramic radiograph showed a large unilocular well defined corticated lesion seen in maxilla crossing the midline extending from maxillary right lateral incisor to the maxillary left 2nd molar (marked by black arrows). Superiorly the lesion is extending to the left infraorbital region causing the superior displacement of the left nasal floor and inferiorly upto the alveolar crestal bone. Overlapping was noted with maxillary right lateral incisor and canine (Fig. 3). The findings on OPG led to the differential diagnosis of radicular cyst, OOC, KCOT. Computed tomography scan report gave an impression of a lytic expansile lesion on the left maxilla causing thinning of its walls at some places. No evidence of enhancement was found on post contrast images. Axial section of the CT scans showing a lytic expansile lesion on the left maxilla causing palatal and mild buccal cortical bone expansion (Fig. 4) (marked by white arrows). Computed tomography scan axial views also showed (A) well-defined corticated hypodense expansile lesion with erosion of the buccal cortical plate near the molar region. (B) The lesion also shows discontinuity in the anterior and medial wall of maxillary sinus (Figs 5A and B) and deviation of the nasal septum toward the right side and erosion of the medial wall of left maxillary sinus (marked by white arrow) (Fig. 6). 


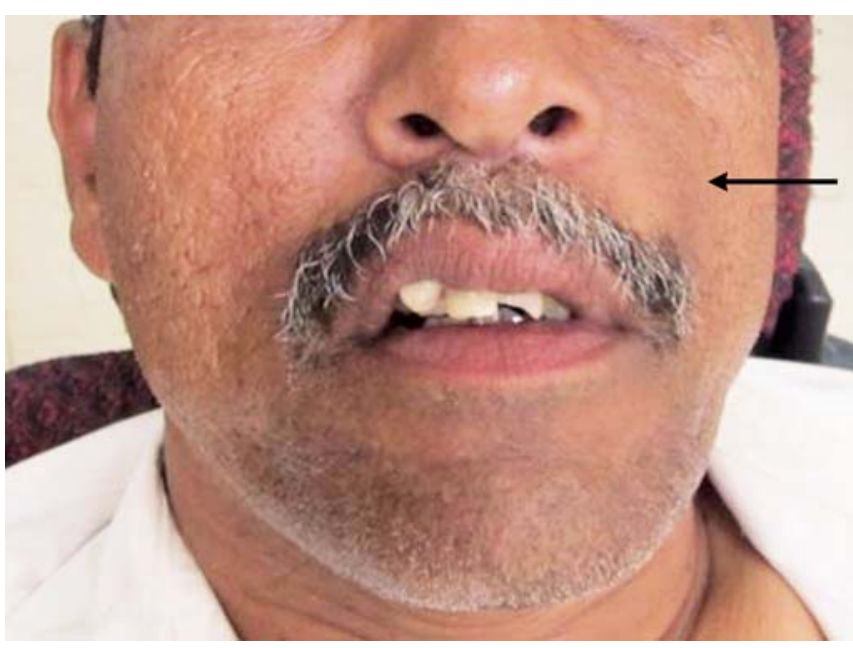

Fig. 1: Anterior facial view of the patient showing a slight swelling on the left side with obliteration of the left nasolabial fold

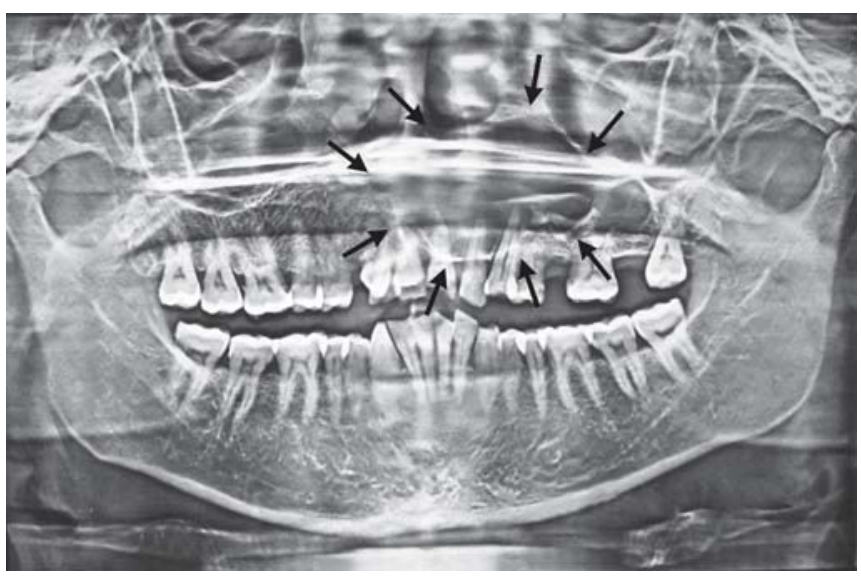

Fig. 3: Panoramic radiograph showing a large unilocular well-defined corticated lesion seen in maxilla crossing the midline extending from maxillary right lateral incisor to the maxillary left 2nd molar (marked by black arrows). Superiorly, the lesion is extending to the left infraorbital region causing the superior displacement of the left nasal floor and inferiorly upto the alveolar crestal bone. Overlapping seen with maxillary right lateral incisor and canine

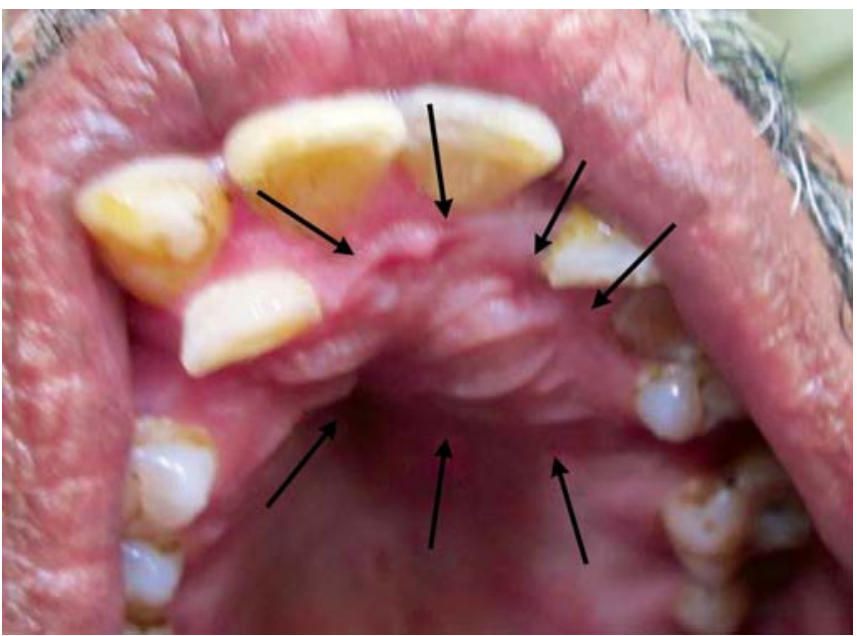

Fig. 2: Intraoral image showing swelling in the left anterior palate region

Computed tomography scan sagittal view showed (A) welldefined corticated hypodense expansile lesion with erosion of the anterior buccal cortical plate and buccal cortical plate near the molar region. (B) Discontinuity in the anterior and medial wall of maxillary sinus perforating its floor (marked by black arrows) (Figs 7A and B). After analyzing the OPG and various CT scans, OOC, KCOT were considered the most likely working diagnosis.

\section{Treatment}

To minimize invasiveness and recurrence, the most effective treatment option appeared to be enucleation of the OOC and subsequent application of Carnoy's solution. Alternatively, marsupialization followed by cystectomy was done, as this treatment does not result in a significantly higher rate of recurrence than enucleation plus Carnoy's solution. Lesions treated in this manner require several

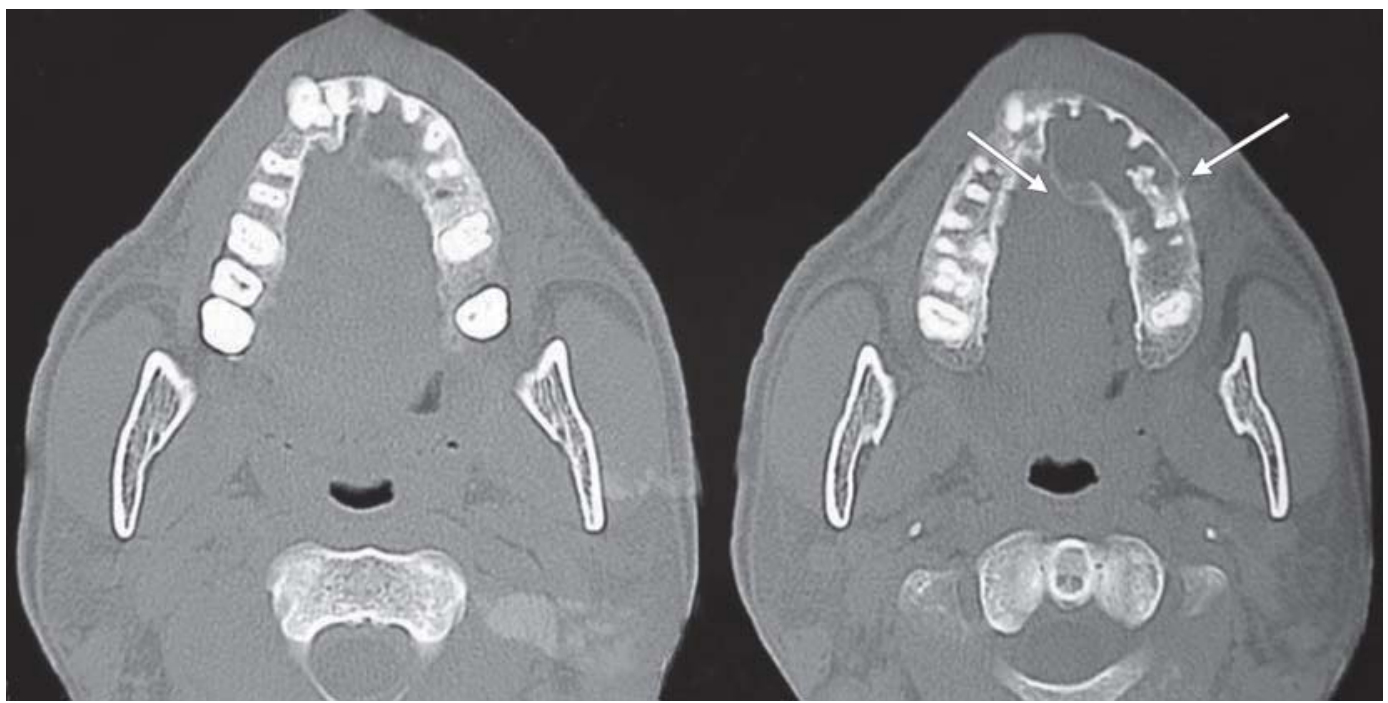

Fig. 4: Axial section of the CT scans showing a lytic expansile lesion on the left maxilla causing palatal and mild buccal cortical bone expansion (marked by white arrows) 


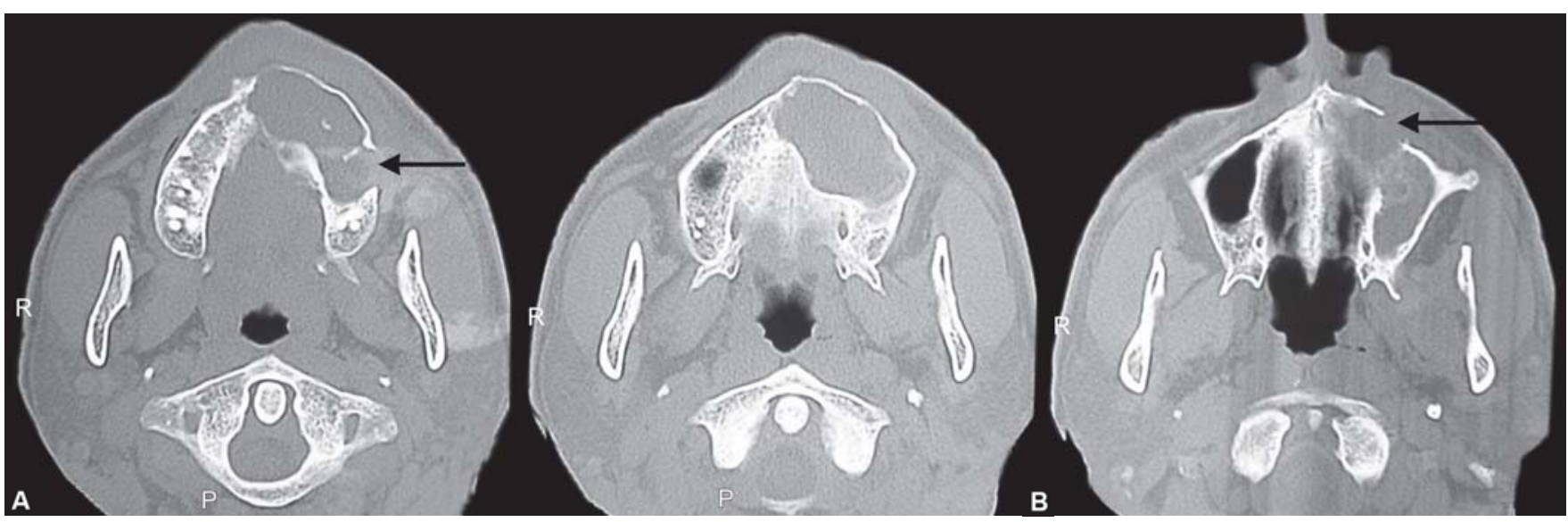

Figs 5A and B: Computed tomography scan axial views showing: (A) well-defined corticated hypodense expansile lesion with erosion of the buccal cortical plate near the molar region, $(B)$ discontinuity in the anterior and medial wall of maxillary sinus (marked by black arrows)
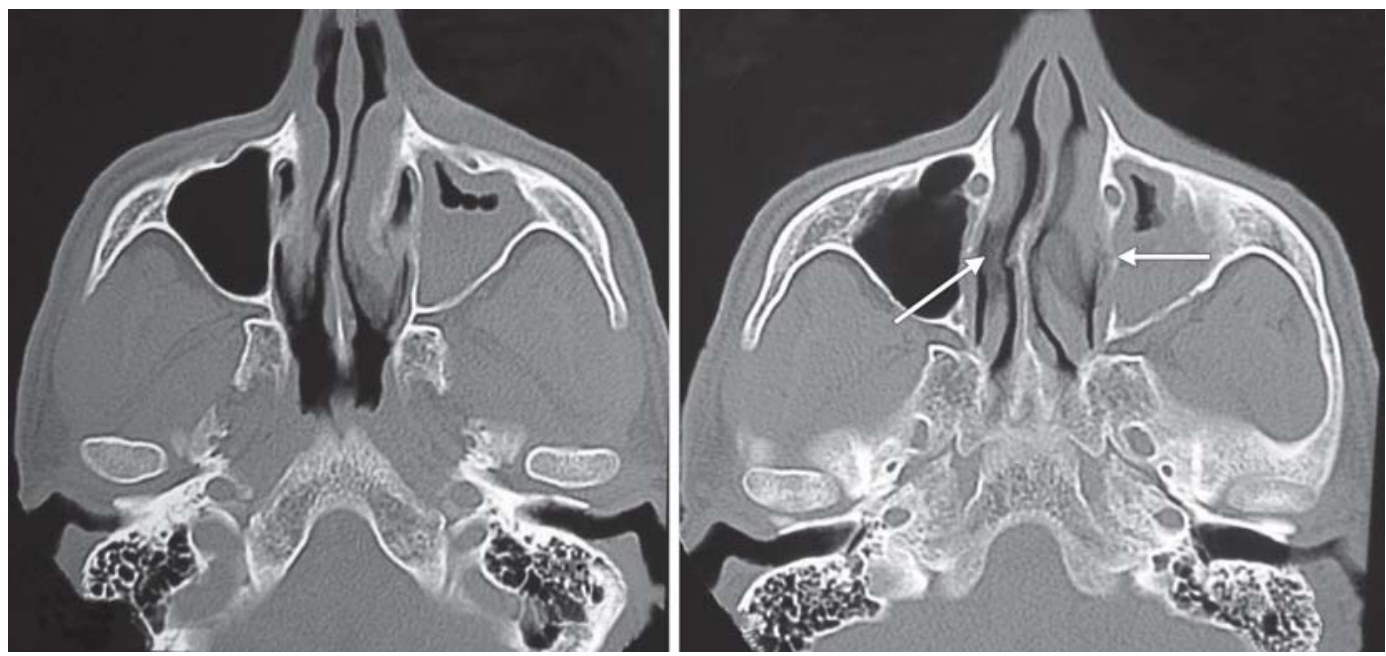

Fig. 6: Computed tomography scan (axial view) showing deviation of the nasal septum toward the right side and erosion of the medial wall of left maxillary sinus (marked by white arrows)

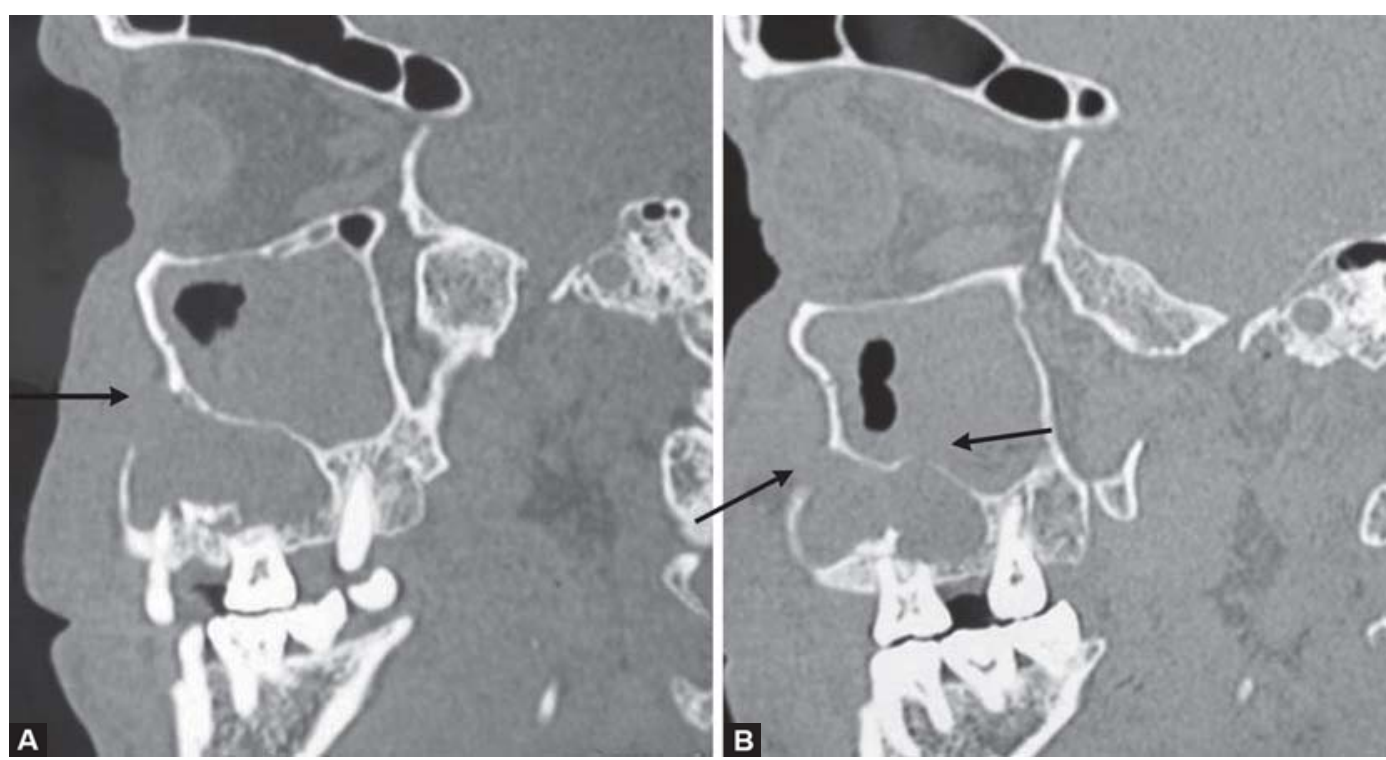

Figs 7A and B: Computed tomography scan (sagittal view) showing: $(A)$ well-defined corticated hypodense expansile lesion with erosion of the anterior buccal cortical plate and buccal cortical plate near the molar region, (B) discontinuity in the anterior and medial wall of maxillary sinus perforating the floor of the maxillary sinus (marked by black arrows) 


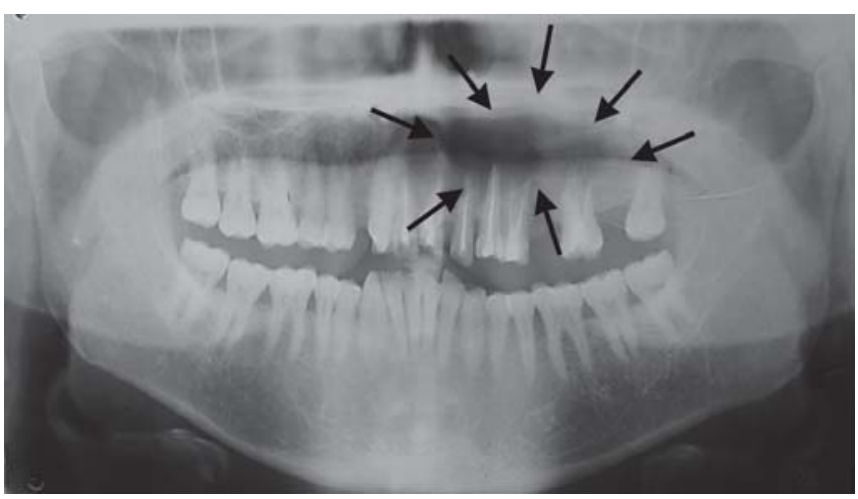

Fig. 8: Postsurgery panoramic radiograph showing radiolucency periapical to root canal treated maxillary right central incisor to the maxillary left first molar crossing the midline suggestive of a healing scar. Mild alveolar bone loss in relation to the maxillary left central and lateral incisors is seen. Missing teeth are maxillary left 2nd premolar and 2nd molar

months of at-home irrigation by the patient as well as clinical observation before enucleation and hence followed. Post surgery panoramic radiograph showed radiolucency periapical to root canal treated maxillary right central incisor to the maxillary left first molar crossing the midline suggestive of a healing scar. Mild alveolar bone loss in relation to the maxillary left central and lateral incisors was seen. Maxillary left 2nd premolar and 2nd molar were missing (Fig. 8).

\section{DISCUSSION}

OOC can be found in both maxilla and mandible with greater predilection for mandible (Table 1). This patient presented with a swelling in the maxilla who gave history of trauma 8 years back. Various radiographs, like panoramic radiograph and CT scan, were taken to locate and judge the extent of the lesion. The panoramic radiograph taken gave a preliminary idea about the lesion, which was large radiolucent unilocular well-defined corticated lesion seen in maxilla crossing the midline extending from maxillary right left central incisor to the maxillary left 1 st molar. It also gave a judgment of the superoinferior extent of the lesion. The CT scan reports gave an impression of a lytic expansile lesion on the left maxilla causing thinning of its walls in some regions. According to White/Pharoah, an important characteristic of the KCOT is its propensity to grow along the internal aspect of the jaw causing minimal expansion. ${ }^{7}$ Hence the most likely diagnosis was OOC. ${ }^{8}$ An incisional biopsy of the lesion reported it to be a keratinizing cystic lesion. In view of all the above findings, a provisional diagnosis of OOC was considered. This diagnosis was confirmed after surgical excision of the lesion by histopathology which showed cystic lumen lined by orthokeratinized stratified squamous epithelium composed of 3 to 4 cell layers covering mature fibrous connective tissue with chronic inflammatory cell infiltrate (Fig. 9). Although, histopathological findings give the final diagnosis, OOC can also be differentiated radiographically from KCOT by its buccolingual or buccopalatal expansion and lesser incidence of root resorption.

\section{CONCLUSION}

1. So far the OOC has not shown root resorption, a feature observed significantly frequently in KCOT. Although OOC presented many of the features observed in the KCOT, such as a predilection for the posterior mandible and multilocularity, buccolingual expansion was frequently observed feature. This expanded the entire mesiodistal extent of the lesion, which was not seen in KCOT.

2. Root resorption was not a feature of the OOC and this differs markedly from KCOT.

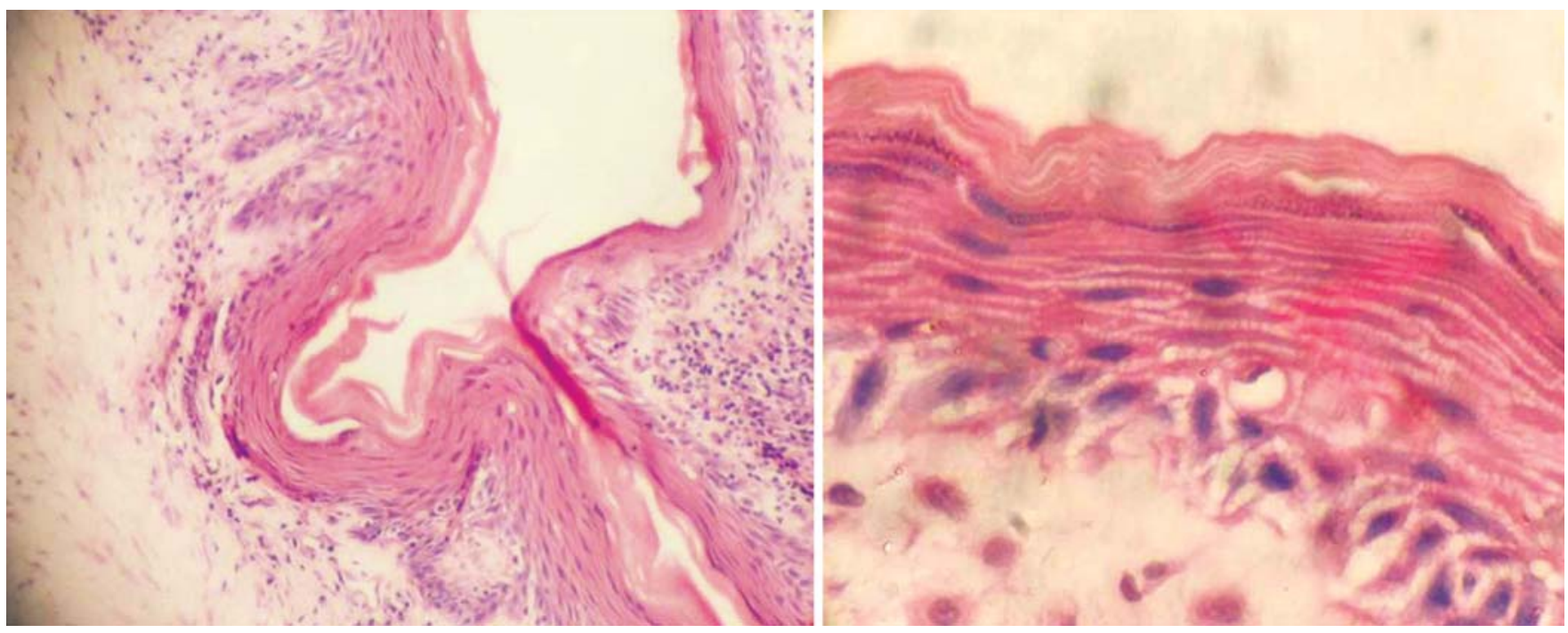

Fig. 9: $\mathrm{H}$ and $\mathrm{E}$ stained section showing cystic lumen lined by orthokeratinized stratified squamous epithelium which is 3 to 4 cell layered can be seen on higher resolution 
Table 1: Comparison between the clinical and radiological features of the OOC and $\mathrm{KCOT}^{7}$

\begin{tabular}{lll}
\hline Feature & $\begin{array}{l}\text { Orthokeratinized } \\
\text { odontogenic cyst }\end{array}$ & $\begin{array}{l}\text { Keratocystic odontogenic } \\
\text { tumor }\end{array}$ \\
\hline Gender, males:females & $2.59: 1$ & $1.42: 1$ \\
Jaw, maxilla:mandible & $9.17: 1$ & $2.08: 1$ \\
Size (mean) in cm & 4.8 & 5.1 \\
Pain & $21.3 \%$ & $5.71 \%$ \\
Expansion, yes:no & $5: 00$ & $27: 06$ \\
Unilocular(\%) & $87 \%$ & $69.4 \%$ \\
Root resorption, yes:no & $0 \%$ & $68.42 \%$ \\
Unerupted teeth(\%) & $60.8 \%$ & $48 \%$ \\
\hline
\end{tabular}

\section{REFERENCES}

1. Philipsen HP. Om keratocystedr (Kolesteratomer) and kaeberne. Tandlaegebladet 1956;60:963-971.

2. Philipsen HP. Keratocystic odontogenic tumour In World Health Organization classification of tumors. Pathology and genetics of head and neck tumors, Barnes L, Eveson JW, Reichart P, Sidranskys D, editors. Lyon: IARC Press, 2005. p. 306-307.

3. Kramer IRH, Pindborg JJ, Shear M. Histological typing of odontogenic tumours. WHO International histological classification of tumours. (2nd ed). London: Springer-Verlag, 1992 p. 35-36.

4. Wright JM. The odontogenic keratocyst: orthokeratinized variant. Oral Surg Oral Med Oral Pathol 1981;51:609-618.

5. Bolbaran V, Martinez B, Rojas R. Odontogenic keratocysts. A retrospective study of 285 cases. (I. Clinical aspects). Med Oral 2000;5:331-337.

6. Bolbaran V, Martinez B, Rojas R. Odontogenic keratocysts. A retrospective study of 285 cases. (II. Histopathological aspects). Med Oral 2000;5:338-344.

7. Dong Q, Pan S, Sun LS, Li TJ. Orthokeratinized odontogenic cyst: A clinicopathologic study of 61 cases. Arch Pathol Lab Med 2010;134:271-275.

8. White SC, Pharoah MJ. Oral radiology: principles and interpretation. 6th ed. St Louis: Mosby; 2008.

\section{ABOUT THE AUTHORS}

\section{Heena Sonawane}

Senior Resident, Department of Oral Medicine and Radiology Nair Hospital Dental College, Mumbai, Maharashtra, India

Correspondence Address: B-404, Surobhi Township, Near Father Michael Society, Tingre Nagar, Pune-411015, Maharashtra, India Phone (Residence): +919890105629, (Work): +91(022)23082714 Ext-209, e-mail: drheenasonawane135@gmail.com

\section{Freny R Karjodkar}

Professor and Head, Department of Oral Medicine and Radiology Nair Hospital Dental College, Mumbai, Maharashtra, India

\section{Kaustubh Sansare}

Associate Professor, Department of Oral Medicine and Radiology Nair Hospital Dental College, Mumbai, Maharashtra, India

\section{Nimish Prakash}

Senior Resident, Department of Oral Medicine and Radiology, Nair Hospital Dental College, Mumbai, Maharashtra, India 